in vivo $32: 1071-1080(2018)$

doi:10.21873/invivo.11348

\title{
Characterisation of a Mouse Model of Breast Cancer with Metabolic Syndrome
}

\author{
LINDA A. BUSS ${ }^{1 *}$, ANISHAH MANDANI ${ }^{1 *}$, ELISABETH PHILLIPS ${ }^{1}$, \\ NICOLA J.A. SCOTT ${ }^{2}$, MARGARET J. CURRIE ${ }^{1}$ and GABI U. DACHS ${ }^{1}$ \\ ${ }^{1}$ Mackenzie Cancer Research Group, Department of Pathology, and ${ }^{2}$ Christchurch Heart Institute, \\ Department of Medicine, University of Otago, Christchurch, New Zealand
}

\begin{abstract}
Background/Aim: Patients with breast cancer and metabolic syndrome have poorer outcomes. We aimed to develop and characterise an apolipoprotein E-null/aromatase knockout (ApoE $\left.E^{-/} / A r K O\right)$ mouse model of breast cancer with metabolic syndrome to aid research of the mechanisms behind poor prognosis. Materials and Methods: Wild-type, ApoE ${ }^{-/-}$ and ApoE ${ }^{-/} / A r K O$ mice were orthotopically implanted with EO771 murine breast cancer cells. Tumour growth was monitored and tumours investigated for pathological features such as cancer-associated adipocytes, hypoxia and cancer cell proliferation. Results: Tumours from ApoE ${ }^{-/-} / A r K O$ mice were significantly more proliferative than those from wild-type mice ( $p=0.003)$, and exhibited reduced expression of insulinlike growth factor binding protein-5 $(p=0.002)$. However, ApoE ${ }^{-/-} /$ArKO mice also had a reduced rate of metastasis compared to wild-type and ApoE ${ }^{-/}$mice. Tumour hypoxia and the number of cancer-associated adipocytes did not differ. Conclusion: The ApoE ${ }^{-/-} / A r K O$ model with EO771 breast cancer provides a novel mouse model to investigate the effects of metabolic syndrome on aspects of breast tumour biology.
\end{abstract}

Metabolic syndrome is characterised by a cluster of disorders that predispose towards development of heart disease, type II diabetes and atherosclerosis (1). An individual is diagnosed as having metabolic syndrome if they have at least three out of five metabolic risk factors: Abdominal obesity, high levels of plasma lipids (hyperlipidaemia), low levels of high-density lipoprotein (HDL) cholesterol, high blood

This article is freely accessible online.

*These Authors contributed equally to this work.

Correspondence to: Associate Professor Gabi U. Dachs, University of Otago Christchurch, 2 Riccarton Ave, Christchurch 8011, New Zealand. E-mail: gabi.dachs@otago.ac.nz

Key Words: Metabolic syndrome, breast cancer, hyperlipidaemia, ApoE, ArKO. pressure (hypertension) and high fasting blood sugar (hyperglycaemia) (1). Epidemiological evidence indicates that individuals with metabolic syndrome also have an increased risk of developing breast cancer (2) and a worse prognosis after diagnosis (3).

Mechanistically, there are a number of ways in which the components of metabolic syndrome can contribute to breast cancer development and progression. Obesity is characterised by chronic, low-grade inflammation, which is reflected by changes in serum levels of inflammatory markers such as interleukin (IL)-6, and growth factors such as insulin-like growth factors (IGFs) and their binding proteins (IGFBPs) $(4,5)$. Chronic inflammation is a known contributor to breast cancer development and progression (6). Obesity-related chronic inflammation is caused by hypoxic, hypertrophic adipocytes and the increased infiltration of pro-inflammatory M1 macrophages into obese adipose tissue (7). Similarly, hyperlipidaemia can lead to the development of chronic inflammation through toll-like receptor signalling on macrophages (8). Cholesterol and its metabolite 27-hydroxycholesterol have been shown to promote tumour cell proliferation, epithelial to mesenchymal transition and accelerated tumour growth and metastasis in mouse models (9-11). In addition, hyperglycaemia has been shown to induce chemoresistance (12) and increase cell migration (13) of oestrogen receptor (ER)-positive breast cancer cells in vitro. Thus, the chronic inflammation and high levels of cholesterol and glucose associated with metabolic syndrome result in more proliferative tumour cells with increased metastatic potential.

It has been shown that adipocytes in close proximity to the tumour become delipidated and interact with tumour cells to stimulate their aggressive behaviour (14). These cells are termed cancer-associated adipocytes (CAA). In vitro, it has been shown that breast cancer cells cultured with adipocytes become more invasive (14). This provides a mechanism whereby excess adipose tissue could directly influence tumour progression, which is of particular importance in breast cancer due to the abundance of adipose tissue in the breast. 
Low oxygen (hypoxia) is a negative prognostic indicator for breast cancer (15). When tumours outgrow their blood supply, regions of hypoxia develop. This activates hypoxiainducible factors (HIF) 1 and 2, transcription factors which regulate cellular adaptation to hypoxia (16). HIF1 and-2 activate a large array of pro-survival, angiogenic and metabolic genes, including vascular endothelial growth factor (VEGF) (16). VEGF is proangiogenic and, under hypoxia, stimulates the formation of new blood vessels. In tumours it is aberrantly expressed, which leads to the formation of dysfunctional vessels and a chaotic vasculature system (17). This results in further perpetuation of tumour hypoxia and the chronic overexpression of HIFs and VEGF. In addition, emerging evidence indicates that obesity and hyperlipidaemia accelerate tumour angiogenesis $(18,19)$.

Apolipoprotein E (ApoE) is a key protein involved in cellular lipid uptake from the bloodstream; thus, ApoE ${ }^{-/-}$ mice are hyperlipidaemic due to impaired lipid clearance from the bloodstream (20). However, they lack other features of metabolic syndrome. The aromatase enzyme is the product of the aromatase cytochrome $P 450$ gene (CYP19) and catalyses the conversion of $\mathrm{C} 19$ androgens to $\mathrm{C} 18$ oestrogens (21). Individuals with a homozygous mutation of the CYP19 gene, resulting in aromatase deficiency, have clinical features of metabolic syndrome, namely insulin resistance and high cholesterol (22). Similarly, aromataseknockout $(A r K O)$ mice exhibit insulin resistance, elevated serum cholesterol and age-progressive obesity (22). Scott et al. developed and characterised a mouse model of metabolic syndrome by crossing hyperlipidaemic $A p o E^{-/-}$mice with ArKO mice (23). They reported that these mice display all the characteristics of metabolic syndrome: The mice are heavier than their wild-type counterparts (likely due to an increase in abdominal fat), have elevated serum cholesterol and triglyceride levels, a lower level of HDL cholesterol, loss of glycaemic control and hypertension (23). In addition, measurement of circulating adipokines revealed an inflammatory state. These metabolic abnormalities were strongly evident from 6 months of age.

Due to the epidemiological evidence indicating a protumour effect of metabolic syndrome, preclinical evidence is required to delineate tumour growth characteristics and composition of the tumour microenvironment in a metabolic syndrome model with breast cancer. Currently, most mouse models of metabolic syndrome either do not exhibit all the characteristics of metabolic syndrome or have impaired leptin signalling, which is not representative of the clinical situation [reviewed in $(24,25)]$. In addition, no other preclinical study has investigated the effect of metabolic syndrome on breast cancer (as far as we are aware). Many studies have used obese models to examine the relationship between obesity and tumour growth [reviewed in (26)], and some of these may also have had other components of metabolic syndrome [e.g. diet-induced models of obesity can also be hyperlipidaemic and have insulin resistance (27)], but this was not documented. For the most part, these studies have shown that obesity increases tumour burden and reduces latency (26).

We aimed to develop a metabolic syndrome model with orthotopic breast cancer (tumours grown in their correct anatomical location and tissue) using the $A p o E^{-/-} / A r K O$ mice developed by Scott et al. (23) and inoculating them with EO771 murine breast cancer cells. Our objective was to characterise tumour growth rate, aspects of the tumour microenvironment, and changes in circulating factors in the metabolic syndrome ApoE $E^{-/-} / A r K O$ compared to hyperlipidaemic $A p o E^{-/-}$and wild-type mice.

\section{Materials and Methods}

Mouse model. Ethical approval for this study was obtained from the University of Otago Animal Ethics Committee (C05/14). International guidelines for animal welfare were strictly followed (28). Mice with three different genotypes were used: Wild-type C57BL/6 $(\mathrm{n}=18)$, ApoE $^{-/-}(\mathrm{n}=18)$ and $\operatorname{ApoE}^{-/-} / \operatorname{ArKO}(\mathrm{n}=12)$. $A p o E^{-/-}$mice were originally generated by Piedrahita et al. (20) and bred in-house from homozygous individuals. ApoE $E^{-/-} / \mathrm{ArKO}$ mice were generated by crossbreeding $A p o E^{-/-}$and $A r K O$ [originally generated by Fisher et al. (29)], with genotype testing as previously described (23). To better mimic the age distribution of most patients with breast cancer, middle-aged female mice (aged 6-12 months) were used. Mice were fed a standard chow diet, kept on a 12:12-h light-dark cycle at a temperature of approximately $22^{\circ} \mathrm{C}$ and housed in groups of up to five.

Tumour model. Mice were injected with $2 \times 10^{5}$ EO771 breast cancer cells in sterile phosphate-buffered saline (PBS) into the 4th mammary fat pad to form orthotopic breast tumours. EO771 are a medullary breast cancer cell line originally derived from a spontaneous tumour in a C57BL/6 mouse (30), kindly gifted by Dr Andreas Moeller (QIMR Berghofer, Australia). Tumour growth was monitored daily by calliper measurement and the volume estimated using the following formula: Tumour volume $=$ width $^{2} \times($ length $/ 2)$.

When tumours reached a maximum ethical size of $600 \mathrm{~mm}^{3}$, mice were injected intraperitoneally with $60 \mathrm{mg} / \mathrm{kg}$ of the hypoxia marker pimonidazole (Hypoxyprobe Inc., Burlington, MA, USA). After $90 \mathrm{~min}$, mice were anaesthetised by isoflurane (Baxter, Deerfield, IL, USA) inhalation and euthanised by cervical dislocation. Mice were euthanised early if the presence of internal metastases was suspected $(n=11)$. The primary tumour, internal metastases, blood and internal organs were removed for analysis. Tumours were halved; one half was formalin fixed and paraffin embedded (FFPE) and the other was frozen at $-80^{\circ} \mathrm{C}$. Blood was centrifuged at $4,000 \times g$ for $10 \mathrm{~min}$ to obtain serum, which was subsequently stored at $-80^{\circ} \mathrm{C}$. All subsequent analyses were blinded to genotype.

Immunohistochemistry. FFPE samples were sectioned at 3-5 $\mu \mathrm{m}$ and pressure cooker antigen retrieval was performed using citrate buffer [10 mM trisodium citrate dihydrate, $0.05 \%$ (v/v) Tween-20, $\mathrm{pH} 6.0$ ] prior to commencement of the staining procedure. The following 

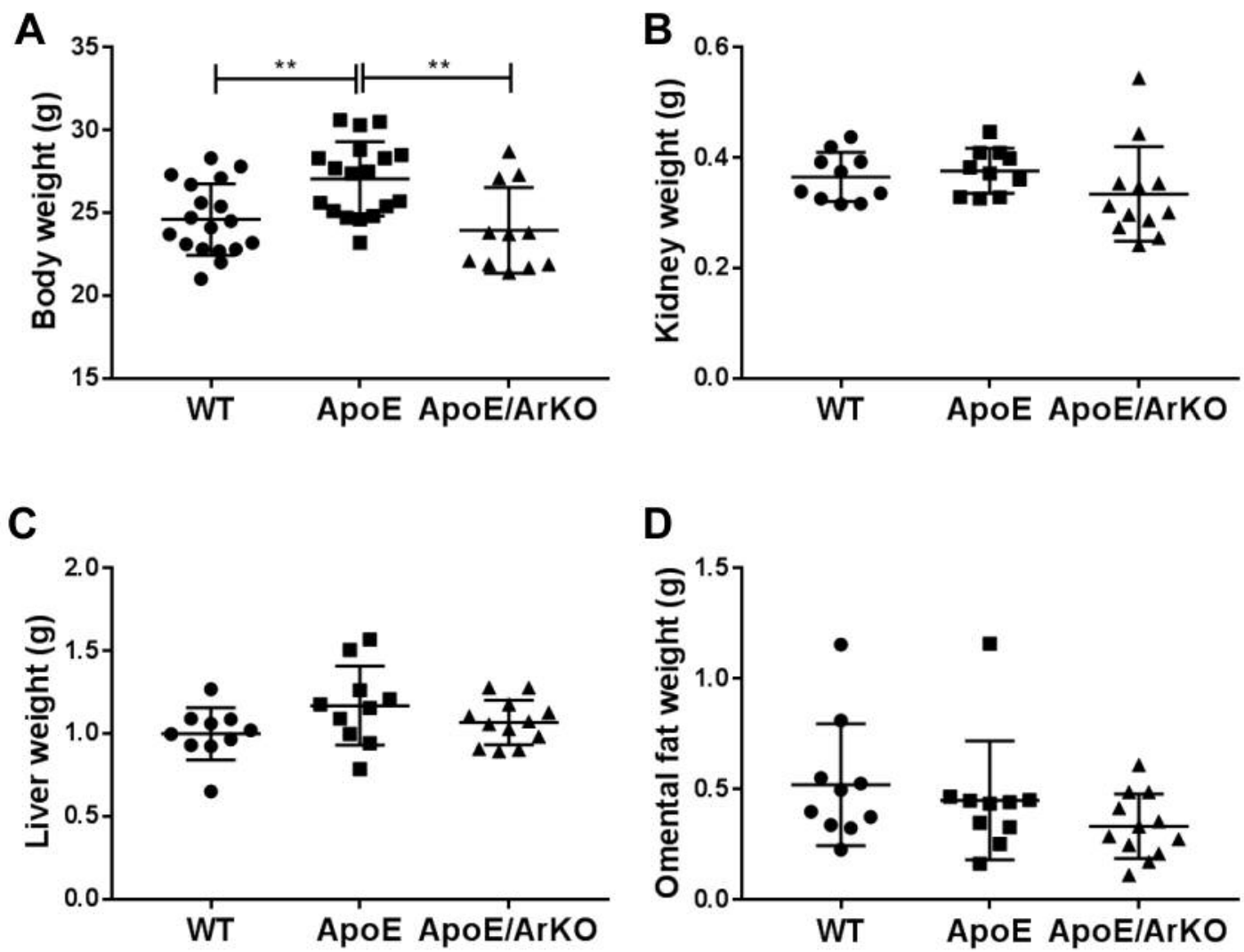

Figure 1. ApoE ${ }^{-/}$mice were significantly heavier than wild-type (WT) and ApoE $E^{-/-} / A r K O$ mice. A: Body weight of wild-type, ApoE ${ }^{-/-}$and ApoE $E^{-/} / A r K O$ mice at the start of the study. Wild-type and ApoE: $n=18$; ApoE/ArKO: $n=11$. Kidney (B), Liver $(C)$ and omental fat $(D)$ weight of wild-type, ApoE $E^{-/-}$and ApoE $E^{-/} /$ArKO mice at euthanasia. Wild-type and ApoE: $n=10 ;$ ApoE/ArKO: $n=12$. **Significantly different at p<0.01. Data are expressed as individual data points and mean $\pm S D$.

antibodies were used for staining: anti-perilipin (1:800, ab3526; Abcam, Melbourne, Australia), anti-phosphohistone H3 (pHH3; 1:100, ab5176; Abcam) and anti-pimonidazole (1:1,000, HP1-1000 kit; Hypoxyprobe Inc.). Antibodies were incubated overnight at $4^{\circ} \mathrm{C}$ with sections, and sections were then stained using the EnVisionTM G/2 System/AP Rabbit/Mouse (Permanent Red) kit (Dako, Copenhagen, Denmark). Perilipin and pHH3 staining was quantified by calculating the percentage of positively stained cells per highpowered field and averaging over 20 random fields. Pimonidazole staining was quantified using a modified $\mathrm{H}$-score by three independent observers (AM, LAB, GUD) as previously described (31). Briefly, staining intensity was assigned a score from 1 (weak) to 3 (strong), and this number was multiplied by the percentage area stained.

Tumour lysates. Frozen tumour samples were chilled using liquid nitrogen and ground into a fine powder using a mortar and pestle on dry ice. Samples were then lysed using ice-cold RIPA buffer $(150 \mathrm{mM} \mathrm{NaCl}, 50 \mathrm{mM}$ Tris, $1 \% \mathrm{NP}-40,0.5 \%$ sodium deoxycholate, $0.1 \%$ sodium dodecyl sulfate, $\mathrm{pH}$ 8.0) with freshly added protease inhibitor cocktail (Roche, Indianapolis, MN, USA), as described previously (31). Samples were sonicated and centrifuged to remove cellular debris before storage at $-80^{\circ} \mathrm{C}$.
Adipokine antibody array. The presence of adipokines in tumour lysates was estimated using an Adipokine Antibody Array (R\&D Systems), following the manufacturer's recommendations. This array simultaneously detects 38 different proteins. Briefly, nitrocellulose membranes were loaded with $400 \mu \mathrm{g}$ of protein samples (a mixture from four mice per group per membrane) and incubated overnight at $4^{\circ} \mathrm{C}$ on a rotating platform. Staining was visualised using UVItec Alliance 4.7 (Cambridge, UK).

Enzyme-linked immunosorbent assay. Serum and tumour IGFBP5, VEGF and IL6 were quantified by enzyme-linked immunosorbent assay (ELISA) using the following kits and according to the manufacturer's instructions: Mouse IGFBP5 ELISA (Abcam), Mouse VEGF DuoSet ELISA (R\&D Systems) and Mouse IL6 Quantikine ELISA Kit (R\&D Systems). Absorbance was measured using a Wallac 1420 Victor $^{3}$ microplate reader (PerkinElmer Life and Analytical Sciences, Waltham, MA, USA).

Statistical analysis. All statistical analyses were performed using GraphPad PRISM 7 (La Jolla, CA, USA). Populations were assessed for normality using the Kolgomorov-Smirnov normality test. Differences across more than two groups were tested using either an ordinary one-way analysis of variance (ANOVA) with Tukey's post- 

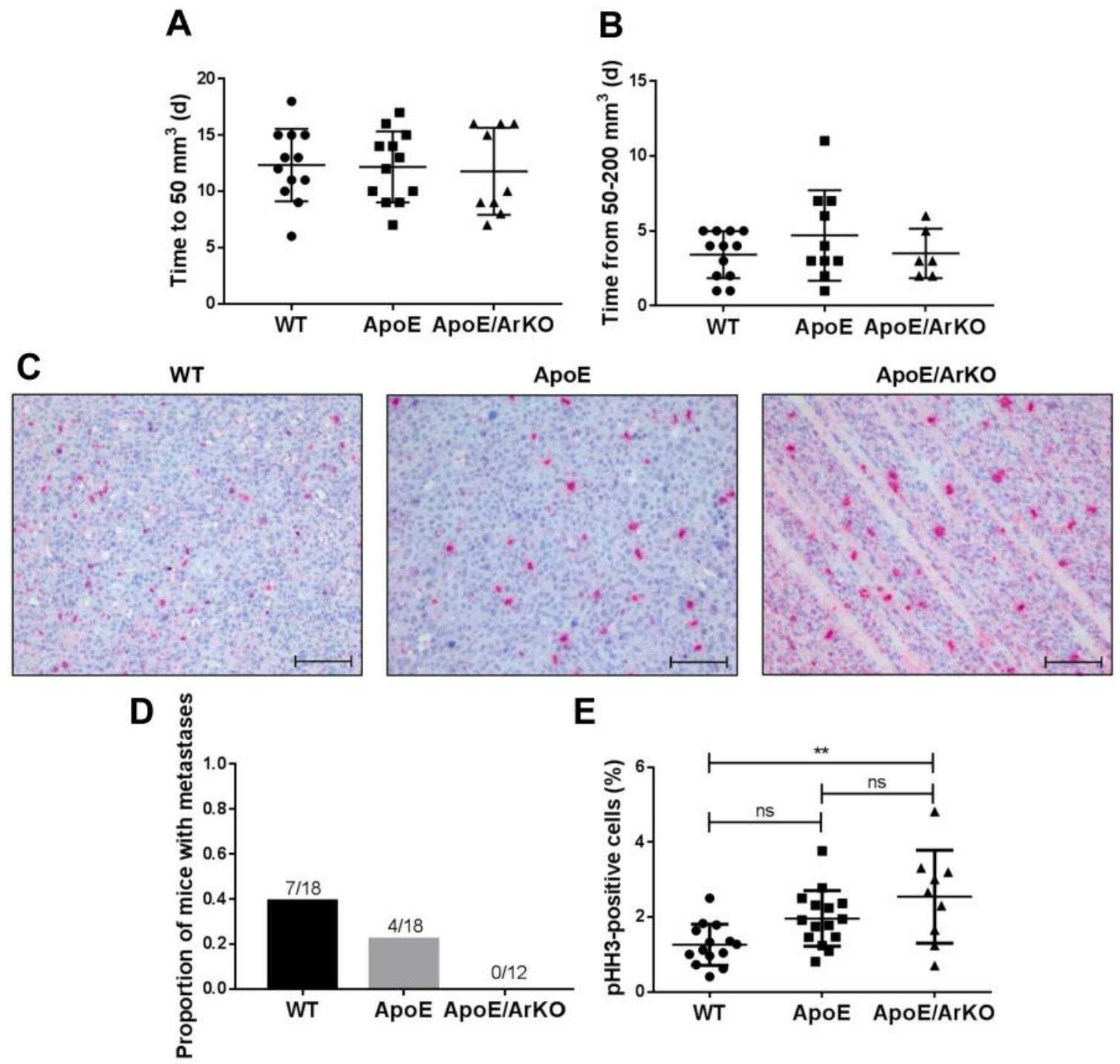

Figure 2. Tumours from ApoE-/-/ArKO mice were significantly more proliferative than tumours from wild-type (WT) mice. A: Analysis of the lag phase of tumour growth as time in days (d) to reach $50 \mathrm{~mm}^{3}$ in wild-type, ApoE-/- and ApoE-/-/ArKO mice. Wild-type and ApoE: n=12; ApoE/ArKO: $n=9$. B: Analysis of the exponential phase of tumour growth as time in days to quadruple in volume. Wild-type: $n=12 ;$ ApoE: $n=10$; ApoE/ArKO: $n=6$. C: Representative immunohistochemical staining for the mitotic marker phosphohistone H3 (pHH3) in tumours from wild-type, ApoE ${ }^{-/-}$and ApoE ${ }^{-/-} / A r K O$ mice. Scale bars represent $100 \mu \mathrm{m}$. D: Proportion of wild-type, ApoE ${ }^{-/-}$and ApoE ${ }^{-/-/ A r K O ~ m i c e ~ w i t h ~ i n t e r n a l ~}$ metastases. E: Analysis of tumour cell proliferation from wild-type, ApoE $E^{-/}$and ApoE $E^{-/} / \mathrm{ArKO}$ mice according to the percentage of pHH3-positive cells. Wild-type: $n=14 ;$ ApoE: $n=15 ;$ ApoE/ArKO: $n=9$. **Significantly different at $p<0.01$, ns: not significant. Data are expressed as individual data points and mean $\pm S D$.

hoc test when data were normally distributed, or a Kruskal-Wallis test with Dunn's post-hoc test when data did not follow a Gaussian distribution. Correlations were performed using Pearson correlation. $p$-Values lower than 0.05 were considered significant.

\section{Results}

Mouse body and organ weight. Hyperlipidaemic ApoE ${ }^{-/-}$ mice were significantly heavier than both wild-type and ApoE $E^{-/-} / A r K O$ mice $(p=0.007$ and $p=0.003$, respectively;
Figure 1A), but there was no significant difference in the body weight of wild-type compared to $A p o E^{-/} / A r K O$ mice. There was no difference in the weights of kidney, liver or omental fat between the three genotypes (Figure 1B-D).

Tumour growth and cancer cell proliferation. Tumour growth rate was investigated by analysing the lag phase of growth, or time taken until tumour establishment $\left(50 \mathrm{~mm}^{3}\right)$, and the exponential phase of growth, or time taken for the 

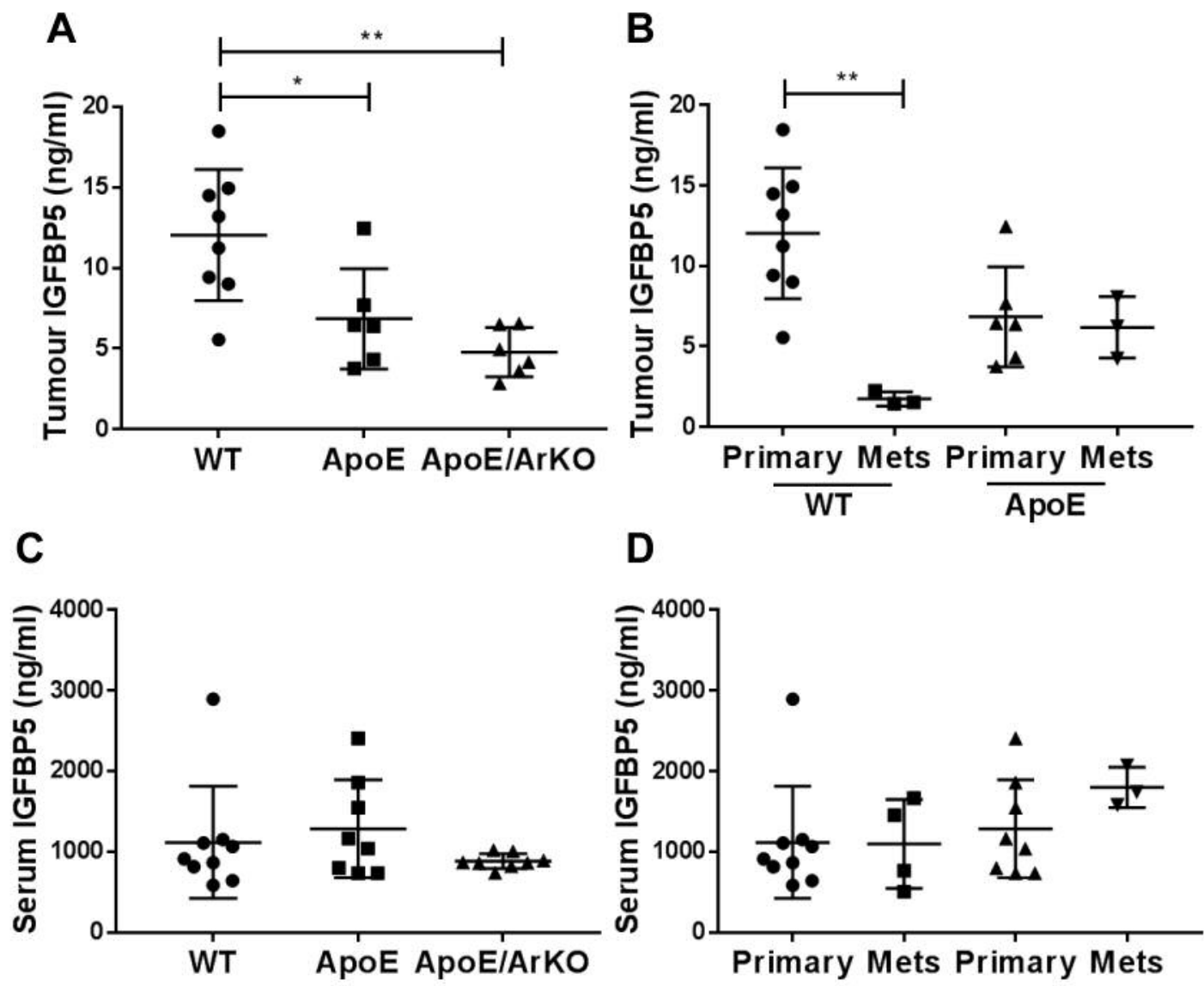

D

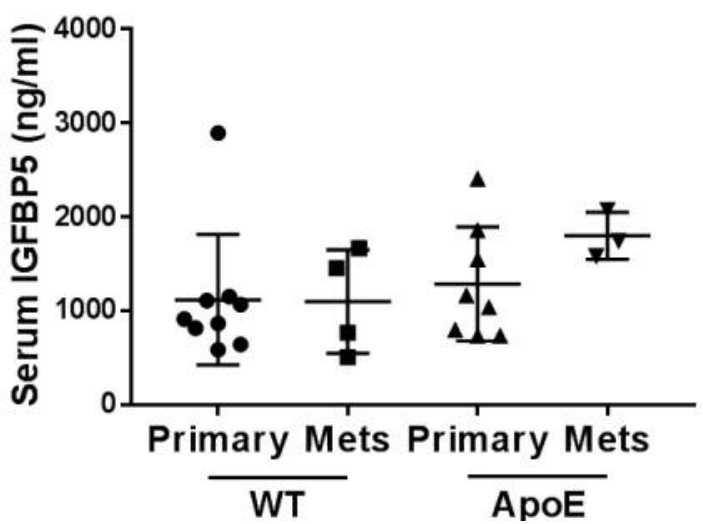

Figure 3. Tumour insulin-like growth factor binding protein 5 (IGFBP5) was reduced in ApoE ${ }^{-/-}$and ApoE $E^{-/-} /$ArKO compared to wild-type (WT) mice. A: Analysis of tumour IGFBP5 in wild-type, ApoE-/- and ApoE-/-/ArKO mice. Wild-type: $n=8$; ApoE and ApoE/ArKO: $n=6$. B: Analysis of IGFBP5 in primary tumours and metastases (Mets) from wild-type and ApoE $E^{-1-}$ mice. Wild-type Primary: $n=8$, Mets $n=3 ;$ and ApoE Primary: $n=6$, Mets: $n=3$. C: Analysis of serum IGFBP5 in wild-type, ApoE ${ }^{-1-}$ and ApoE $E^{-/} /$ArKO mice. Wild-type: $n=9 ;$ ApoE and ApoE/ArKO: $n=8$. D: Analysis of serum IGFBP5 in wild-type and ApoE ${ }^{-/}$mice bearing only primary tumours or with internal metastases. Wild-type Primary: $n=9$, Mets: $n=4$; ApoE Primary: $n=8$, Mets: $n=3$. Significantly different at $* p<0.05$, and ${ }^{* *} p<0.01$. Data are expressed as individual data points and mean $\pm S D$.

tumour to quadruple in volume (from 50 to $200 \mathrm{~mm}^{3}$ ). No differences between groups in either the lag or exponential phase of tumour growth were observed (Figure 2A and B).

Tumour cell proliferation, as measured by the mitotic marker pHH3, was significantly higher in tumours from $A p o E^{-/-} / A r K O$ mice compared to wild-type mice ( $p=0.003$, Figure $2 \mathrm{E}$ ). There was a trend for tumours from $A p o E^{-/-}$ mice to be more proliferative than those from wild-type mice ( $p=0.07$ ), but no difference was observed between $A p o E^{-/-}$ and $A p o E^{-/-} / A r K O$ mice.

A proportion of mice developed ascites and highly aggressive metastases within the peritoneal cavity. Wild-type mice were most prone to these secondary tumours, with $39 \%$ (7/18) developing internal tumours (Figure 2D). In contrast, $22 \%$ (4/18) of $A p o E^{-/-}$mice developed internal tumours, and $0 \%(0 / 12)$ of $A p o E^{-/-} / A r K O$ mice did.
Adipokines in tumours and serum. The antibody array screen detected 23 out of 38 proteins on the adipokine array, with four proteins found to be increased and seven reduced by more than $25 \%$ in tumours from $A p o E^{-/-}$and $A p o E^{-/-} / A r K O$ compared to wild-type mice (results not shown). IGFBP5 and VEGF changed most notably in tumours from $A p o E^{-/-} / A r K O$ compared to wild-type mice and were analysed further by ELISA: In addition, IL-6 was measured as an inflammatory marker.

Tumour IGFBP-5 was significantly reduced in $\mathrm{ApoE}^{-/-}$ $(p=0.02)$ and $A p o E^{-/-} / A r K O$ compared to wild-type mice $(p=0.002$, Figure $3 \mathrm{~A})$. In tumours from wild-type mice, IGFBP5 levels were significantly lower in internal metastases compared to primary tumours $(p=0.001$, Figure $3 \mathrm{~B})$. This difference between primary and secondary tumours was not detected in $A p o E^{-/-}$mice; $A p o E^{-/-} / A r K O$ mice did 


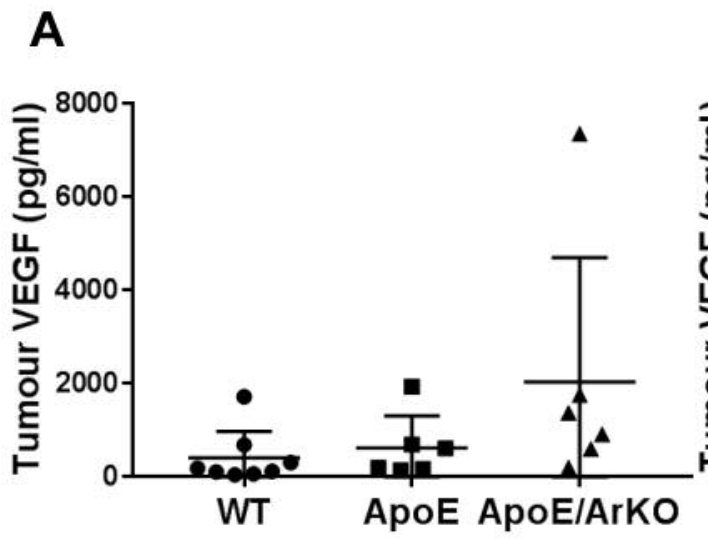

B
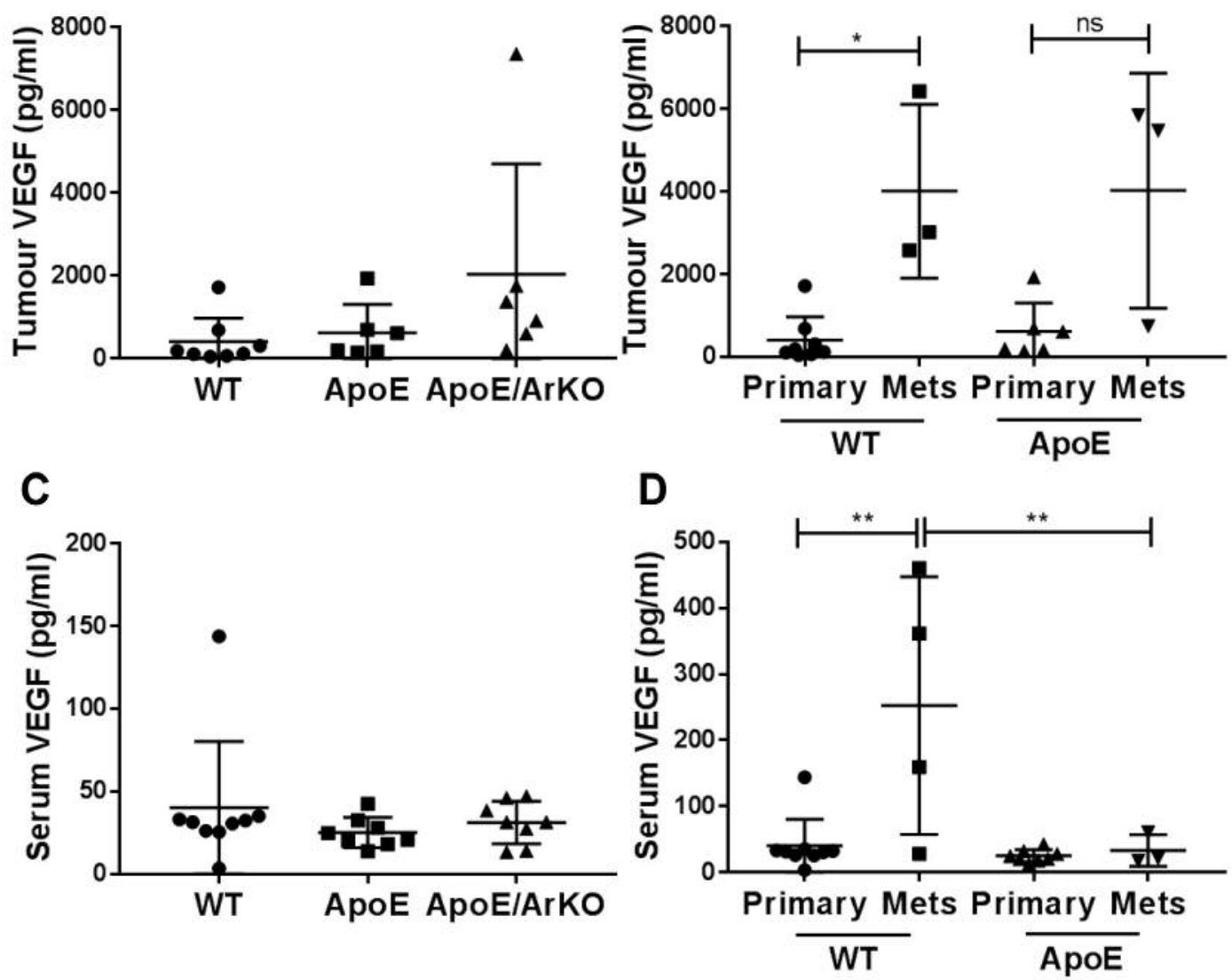

Figure 4. Tumour and serum vascular endothelial growth factor (VEGF) was increased in wild-type (WT) mice with internal metastases compared to wild-type mice bearing only primary tumours. A: Analysis of tumour VEGF in wild-type, ApoE-/- and ApoE-/-/ArKO mice. Wild-type: n=8; ApoE and ApoE/ArKO: $n=6$. B: Analysis of VEGF in primary tumours and metastases from wild-type and ApoE ${ }^{-/-}$mice. Wild-type Primary: $n=8$, Mets: $n=3$; and ApoE Primary: $n=6$, Mets: $n=3$. C: Analysis of serum VEGF in wild-type, ApoE ${ }^{-/-}$and ApoE ${ }^{-/-/ A r K O ~ m i c e . ~ W i l d-t y p e: ~ n=9 ; ~}$ ApoE and ApoE/ArKO: $n=8$. D: Analysis of serum VEGF in wild-type and ApoE ${ }^{-/}$mice bearing only primary tumours or with internal metastases. Wild-type Primary: $n=9$, Mets: $n=4$; and ApoE Primary: $n=8$, Mets: $n=3$. Significantly different at $* p<0.05$, and $* * p<0.01$; ns: not significant. Data are expressed as individual data points and mean $\pm S D$.

not develop visible metastases in this study. Serum IGFBP5 did not differ between groups (Figure 3C) nor between mice with only a primary tumour compared to those with metastases (Figure 3D).

There was no significant difference in tumour or serum VEGF between groups (Figure 4A and C). However, in wildtype mice, internal metastases had significantly higher levels of VEGF compared to primary tumours $(p=0.03)$; this trend was mirrored in tumours from $A p o E^{-/-}$mice, although it was not statistically significant (Figure 4B). Serum levels of VEGF were significantly higher in wild-type mice with internal metastases compared to mice with primary tumours only ( $p=0.001$, Figure 4D), but this was not seen in $A p o E^{-/-}$mice. In mice with internal metastases, serum VEGF was significantly lower in $A p o E^{-/-}$mice compared to wild-type mice $(p=0.009)$.
Levels of tumour and serum IL6 remained unchanged in all groups (Figure 5A and C). No difference was seen in IL6 levels in secondary compared to primary tumours (Figure $5 B$ ), and serum levels were unchanged in mice with metastases compared to those with primary tumours only (Figure 5D). There was a non-significant trend for tumourbearing mice to have elevated serum IL6 compared to nontumour-bearing mice (Figure 5C).

Adipocyte infiltration and tumour hypoxia. There were a notable number of adipocytes infiltrating EO771 orthotopic tumours. However, there was no difference in the number of CAA within the tumours of $A p o E^{-/-}$or $A p o E^{-/-} / A r K O$ compared to wild-type mice (Figure $6 \mathrm{~A}$ and $\mathrm{B}$ ). In addition, there was no difference in the number of CAA in internal 
A
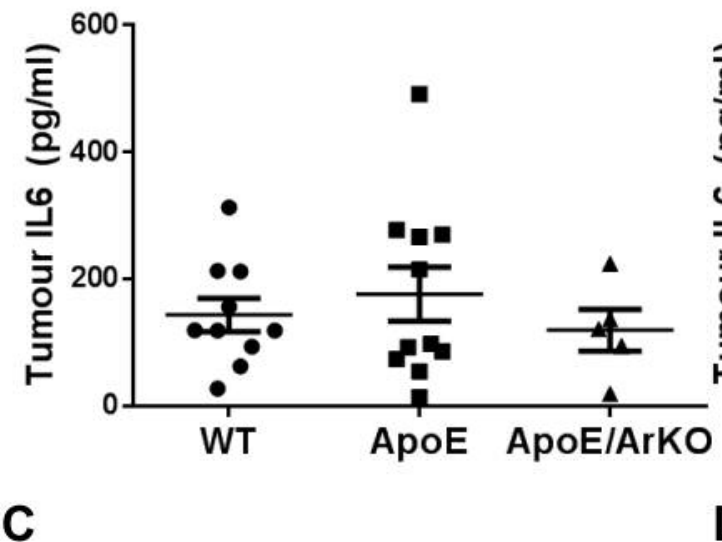

B
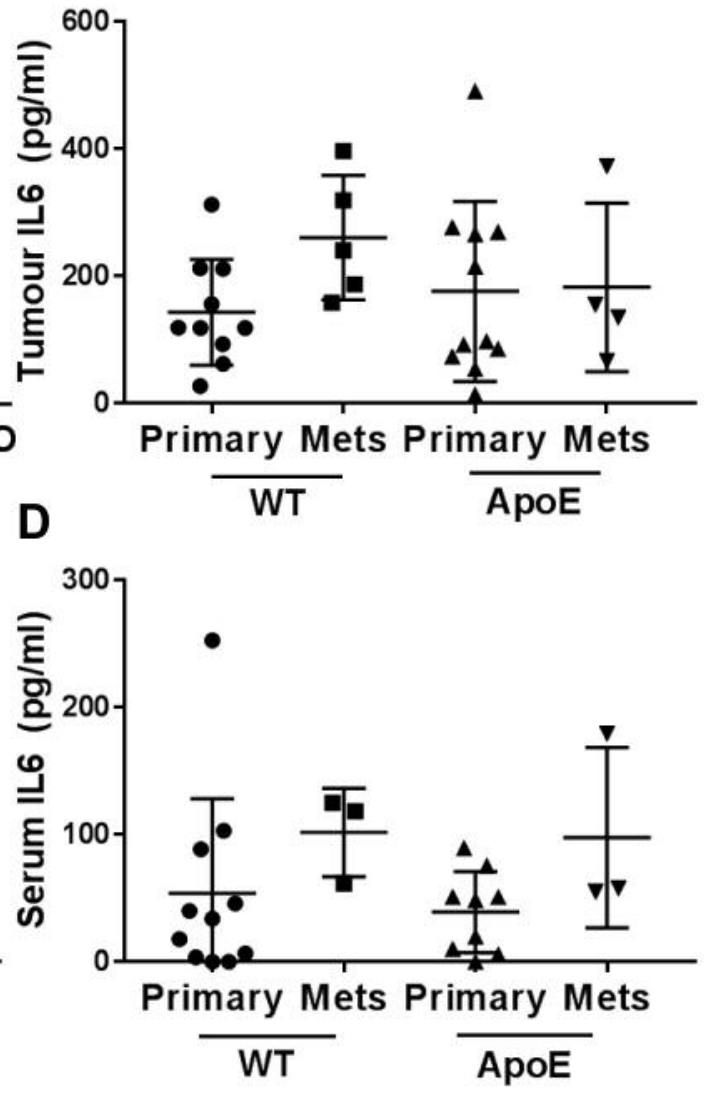

Figure 5. Tumour and serum interleukin 6 (IL6) levels did not differ in ApoE ${ }^{-/-}$and ApoE $E^{-/-/ A r K O ~ c o m p a r e d ~ t o ~ w i l d-t y p e ~(W T) ~ m i c e . ~ A: ~ A n a l y s i s ~}$ of tumour IL6 in wild-type, ApoE-/- and ApoE-/-/ArKO mice. Wild-type: $n=10 ;$ ApoE: $n=11$; ApoE/ArKO: $n=5$. B: Analysis of IL6 in primary tumours and metastases from wild-type and ApoE ${ }^{-1-}$ mice. Wild-type Primary: $n=10$, Mets: $n=5$; and ApoE Primary: $n=11$, Mets: $n=4$. C: Analysis of serum IL6 in non-tumour-bearing (NT) and tumour-bearing (T) wild-type, ApoE ${ }^{-/}$and ApoE-/-/ArKO mice. Wild-type (NT) and ApoE (NT): n=3; ApoE/ArKO (NT): n=4; wild-type (T): n=11; ApoE (T): n=9; ApoE/ArKO (T): $n=8$. D: Analysis of serum IL6 in wild-type and ApoE-/- mice bearing only primary tumours or with internal metastases. Wild-type Primary: $n=11$, Mets $n=3$; and ApoE Primary: $n=9$, Mets: $n=3$. Data are expressed as individual data points and mean $\pm S D$.

metastases compared to primary tumours for either wildtype or $A p o E^{-/-}$mice, nor was there a difference in CAA between metastases from wild-type compared to $\mathrm{ApoE}^{-/-}$ mice (Figure 6C).

The level of tumour hypoxia was similar between groups (Figure 7A and B). However, in $A p o E^{-/-}$mice, metastases were significantly more hypoxic than primary tumours $(p<0.0001)$ (Figure 7C). Interestingly, we observed no difference in the level of hypoxia in primary tumours compared to metastases from wild-type mice. Metastases from $A p o E^{-/-}$mice were significantly more hypoxic than metastases from wild-type mice $(p<0.0001)$ (Figure 7C).

\section{Discussion}

In this study, we aimed to develop and characterise a mouse model of breast cancer with metabolic syndrome. Tumours from $A p o E^{-/} / A r K O$ mice were more proliferative and had reduced levels of tumour IGFBP5 compared to wild-type mice, but showed no difference in the number of tumourinfiltrating adipocytes, level of tumour hypoxia, serum IGFBP5, or serum and tumour VEGF or IL6.

Omental fat and organ weights were similar across all three genotypes, with $A p o E^{-/-}$mice about $10 \%$ heavier than wild-type and $A p o E^{-/-} / A r K O$ (by $2.5 \pm 2.3 \mathrm{~g}$ and $3.1 \pm 2.3 \mathrm{~g}$, respectively). In contrast to Scott et al. (23), we found no 

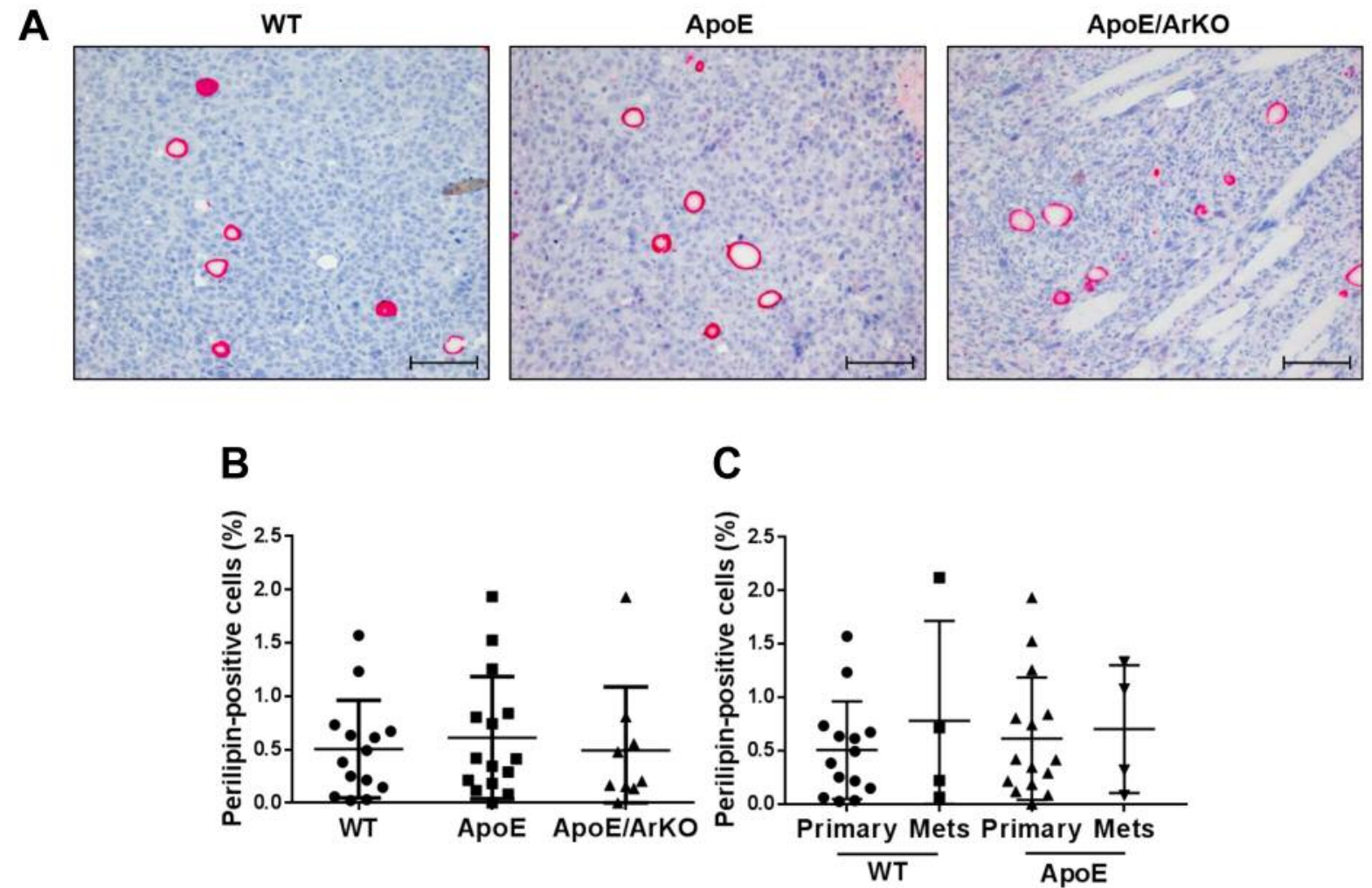

Figure 6. The proportion of cancer-associated adipocytes (CAA) within EO771 tumours did not differ between wild-type, ApoE $E^{-/-}$and ApoE $E^{-/-} / A r K O$ mice. A: Representative immunohistochemical staining for perilipin in tumours from wild-type, ApoE ${ }^{-/}$and ApoE ${ }^{-/-} / A r K O$ mice. Scale bars represent $100 \mu \mathrm{m} . \mathrm{B}$ : Analysis of CAA content in tumours from wild-type, ApoE $E^{-/}$and $A p o E^{-/-} / A r K O$ mice according to the percentage of perilipin-positive cells. Wild-type: $n=14 ;$ ApoE: $n=15$; ApoE/ArKO: $n=9$. C: Analysis of CAA content in primary tumours and metastases from wild-type and ApoE-lmice. Wild-type Primary: $n=14$, Mets: $n=4$; and ApoE Primary: $n=15$, Mets: $n=4$. Data are expressed as individual data points and mean $\pm S D$.

difference in the body weight or omental fat weight of $A p o E^{-/-} / A r K O$ mice compared to wild-type mice. This could be due to the fact that female mice have a weaker weight phenotype than male mice (unpublished observation).

Tumours grown in $A p o E^{-/-} / A r K O$ mice showed significantly higher cancer cell proliferation compared to wild-type mice, with a similar trend observed in $A p o E^{-/-}$ mice. Cholesterol and triglycerides are raised in $\mathrm{ApoE}^{-/-}$and ApoE $E^{-/-} / A r K O$ mice $(23,32)$, and $A p o E^{-/-} / A r K O$ mice also have increased fasting glucose levels (23). It has been shown that cholesterol and high glucose can increase breast tumour cell proliferation $(10,33)$. This suggests that the metabolic abnormalities found in $A p o E^{-/-} / A r K O$ mice enhanced tumour cell proliferation.

The aromatase knockout in $A p o E^{-/-} / A r K O$ mice reduces circulating oestradiol levels (29), which would be expected to reduce tumour growth of $\mathrm{ER}^{+}$tumours. EO771 cells have been reported to be $\mathrm{ER}^{+}(34)$, but in our study no ER staining was detected in the tumours (data not shown). Indeed, EO771 tumours grew at similar rates in all three genotypes.
Tumour levels of IGFBP5 were reduced in $A p o E^{-/-} / \mathrm{ArKO}$ and $A p o E^{-/-}$mice compared to wild-type, with no changes observed in serum. IGFBP5 is involved in the induction of apoptosis in the mammary gland after weaning and may act in a similar manner on breast tumour cells to induce apoptosis (35). Its exact role in breast cancer remains unclear, but it may be dependent on its subcellular localisation $(36,37)$, which our ELISA would not have shown.

Serum IL6 is often elevated in a chronic disease state, such as metabolic syndrome or cancer, and can reflect underlying inflammation (38). In contrast to Scott et al. (23), we saw no difference in serum IL6 in $A p o E^{-/-} / A r K O$ compared to wild-type mice, possibly because circulating IL6 was already elevated by tumour burden.

Other mouse models of metabolic syndrome do not exhibit all the characteristics of metabolic syndrome (25), whereas $A p o E^{-/-} / \mathrm{ArKO}$ mice are reported to display most components (23). However, there are a number of limitations associated with using these mice for cancer research, including minimal 

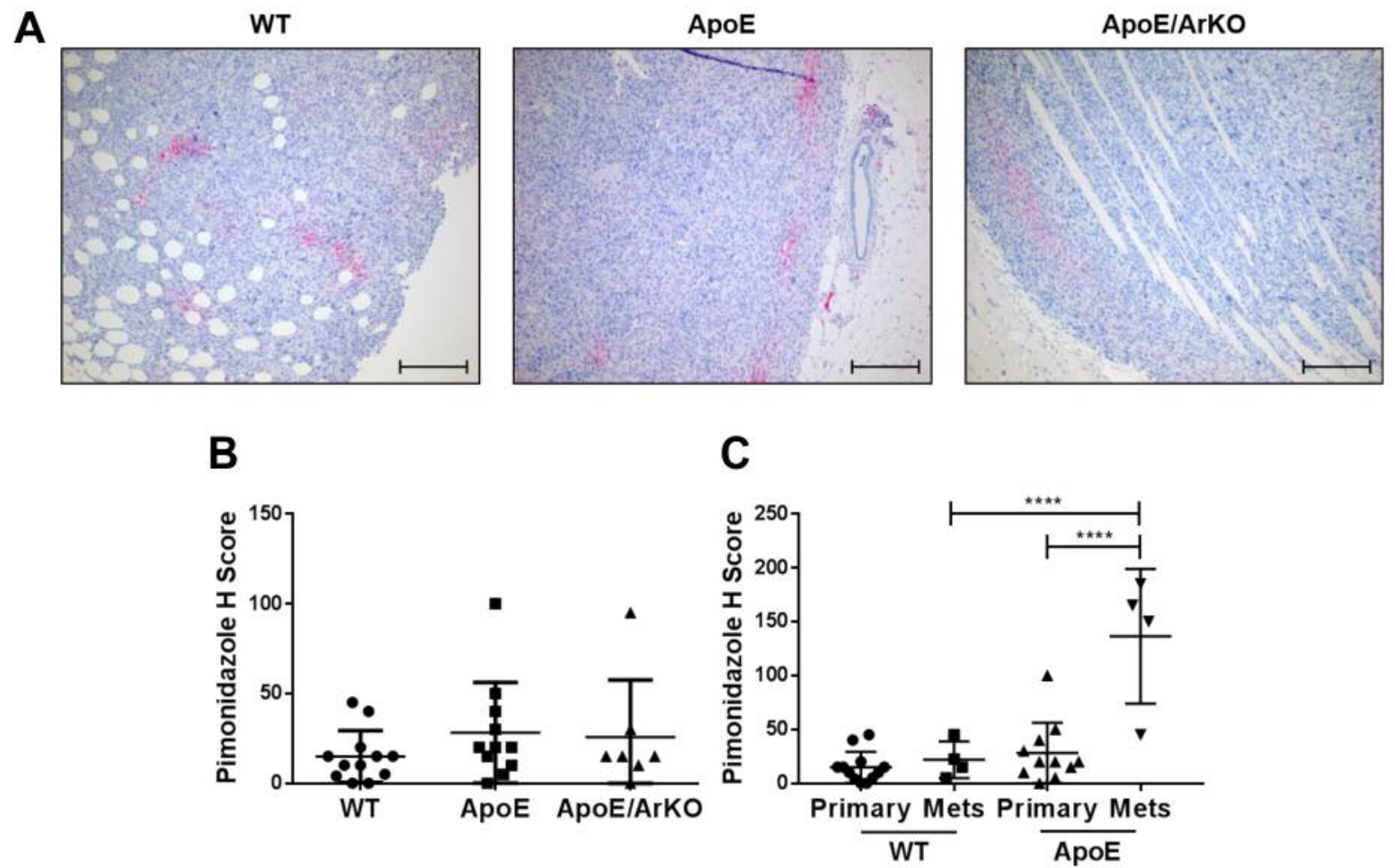

Figure 7. Hypoxia was significantly increased in internal metastases compared to primary tumours from ApoE-/- mice. A: Representative immunohistochemical staining for pimonidazole in tumours from wild-type, ApoE $E^{-/}$and ApoE $E^{-/} / \mathrm{ArKO}$ mice. Scale bars represent $200 \mu \mathrm{\mu m}$. B: Quantification of hypoxia in tumours from wild-type, ApoE ${ }^{-1-}$ and ApoE $E^{-/} /$ArKO mice. Intensity and percentage of staining were quantified by $H$ score. Wild-type: $n=12 ;$ ApoE: $n=11 ;$ ApoE/ArKO: $n=7$. C: Quantification of hypoxia in primary tumours and metastases from wild-type and ApoE ${ }^{-/}$mice. Wild-type Primary: $n=12$, Mets: $n=4$; and ApoE Primary: $n=11$, Mets: $n=4$. $* * * *$ Significantly different at $p<0.0001$. Data are expressed as individual data points and mean $\pm S D$.

signs of abdominal obesity, aromatase deficiency and reduced cellular lipid uptake due to ApoE deficiency.

Our data provide further insight into the role of several components of the metabolic syndrome in breast cancer, specifically cancer cell proliferation and, potentially, metastasis. However, we also highlight the complexities of using genetic knockout models to investigate multifaceted diseases such as metabolic syndrome and breast cancer.

\section{Acknowledgements}

The Authors would like to thank Dr. Leigh Ellmers for maintaining the $A p o E^{-/-}$mouse colony, Dr Andreas Moeller (QIMR Berghofer, Australia) for the EO771 cells, and Dr. Stephen Jamieson (University of Auckland) for training on orthotopic tumour implantation. The Authors appreciate funding from the Mackenzie Charitable Foundation (MJC, EP, GUD).

\section{References}

1 Huang PL: A comprehensive definition for metabolic syndrome. Dis Model Mech 2(5-6): 231-237, 2009.
2 Agnoli C, Grioni S, Sieri S, Sacerdote C, Ricceri F, Tumino R, Frasca G, Pala V, Mattiello A, Chiodini P, Iacoviello L, De Curtis A, Panico S and Krogh V: Metabolic syndrome and breast cancer risk: A case-cohort study nested in a multicentre Italian cohort. PLoS One 10(6): e0128891, 2015.

3 Berrino F, Villarini A, Traina A, Bonanni B, Panico S, Mano MP, Mercandino A, Galasso R, Barbero M, Simeoni M, Bassi MC, Consolaro E, Johansson H, Zarcone M, Bruno E, Gargano G, Venturelli E and Pasanisi P: Metabolic syndrome and breast cancer prognosis. Breast Cancer Res Treat 147(1): 159-165, 2014.

4 Cohen DH and LeRoith D: Obesity, type 2 diabetes and cancer: The insulin and IGF connection. Endocr Relat Cancer 19(5): F27-45, 2012.

5 Mauer J, Denson JL and Brüning JC: Versatile functions for IL-6 in metabolism and cancer. Trends Immunol 36(2): 92-101, 2015.

6 Bhatelia K, Singh K and Singh R: TLRs: Linking inflammation and breast cancer. Cell Signal 26(11): 2350-2357, 2014.

7 Wagner M, Samdal Steinskog ES and Wiig H: Adipose tissue macrophages: The inflammatory link between obesity and cancer? Expert Opin Ther Targets 19(4): 527-538, 2015.

8 Hovland A, Jonasson L, Garred P, Yndestad A, Aukrust P, Lappegard KT, Espevik T and Mollnes TE: The complement system and toll-like receptors as integrated players in the pathophysiology of atherosclerosis. Atherosclerosis 241(2): 480-494, 2015. 
9 Torres CG, Ramí Rez ME, Cruz P, Epuñan MJ, Valladares LE and Sierralta WD: 27-Hydroxycholesterol induces the transition of MCF7 cells into a mesenchymal phenotype. Oncol Rep 26(2): 389-397, 2011

10 Alikhani N, Ferguson RD, Novosyadlyy R, Gallagher EJ, Scheinman EJ, Yakar S and LeRoith D: Mammary tumor growth and pulmonary metastasis are enhanced in a hyperlipidemic mouse model. Oncogene 32(8): 961-967, 2013.

11 Nelson ER, Wardell SE, Jasper JS, Park S, Suchindran S, Howe MK, Carver NJ, Pillai R V, Sullivan PM, Sondhi V, Umetani M, Geradts J and McDonnell DP: 27-Hydroxycholesterol links hypercholesterolemia and breast cancer pathophysiology. Science 342(6162): 1094-1098, 2013.

12 Zeng L, Zielinska HA, Arshad A, Shield JP, Bahl A, Holly JMP and Perks CM: Hyperglycaemia-induced chemoresistance in breast cancer cells: Role of the estrogen receptor. Endocr Relat Cancer 23(2): 125-134, 2016

13 Takatani-Nakase T, Matsui C, Maeda S, Kawahara S and Takahashi K: High glucose level promotes migration behavior of breast cancer cells through zinc and its transporters. PLoS One 9(2): e90136, 2014

14 Dirat B, Bochet L, Dabek M, Daviaud D, Dauvillier S, Majed B, Wang YY, Meulle A, Salles B, Le Gonidec S, Garrido I, Escourrou G, Valet $\mathrm{P}$ and Muller C: Cancer-associated adipocytes exhibit an activated phenotype and contribute to breast cancer invasion. Cancer Res 71(7): 2455-2465, 2011.

15 Semenza GL: The hypoxic tumor microenvironment: A driving force for breast cancer progression. Biochim Biophys Acta - Mol Cell Res 1863(3): 382-391, 2016.

16 Schofield CJ and Ratcliffe PJ: Oxygen sensing by HIF hydroxylases. Nat Rev Mol Cell Biol 5(5): 343-354, 2004.

17 Saharinen P, Eklund L, Pulkki K, Bono P and Alitalo K: VEGF and angiopoietin signaling in tumor angiogenesis and metastasis. Trends Mol Med 17(7): 347-362, 2011.

18 Pelton K, Coticchia CM, Curatolo AS, Schaffner CP, Zurakowski D, Solomon KR and Moses MA: Hypercholesterolemia induces angiogenesis and accelerates growth of breast tumors in vivo. Am J Pathol 184(7): 2099-2110, 2014.

19 Chen C-T, Du Y, Yamaguchi H, Hsu J-M, Kuo H-P, Hortobagyi GN and Hung M-C: Targeting the IKK $\beta / \mathrm{mTOR} / \mathrm{VEGF}$ signaling pathway as a potential therapeutic strategy for obesity-related breast cancer. Mol Cancer Ther 11(10): 2212-2221, 2012.

20 Piedrahita JA, Zhang SH, Hagaman JR, Oliver PM and Maeda $\mathrm{N}$ : Generation of mice carrying a mutant apolipoprotein $\mathrm{E}$ gene inactivated by gene targeting in embryonic stem cells. Proc Natl Acad Sci USA 89(10): 4471-4475, 1992.

21 Meinhardt $U$ and Mullis PE: The essential role of the aromatase/P450arom. Semin Reprod Med 20(3): 277-284, 2002.

22 Jones MEE, Chin Boon W, Proietto J and Simpson ER: Of mice and men: The evolving phenotype of aromatase deficiency. Trends Endocrinol Metab 17(2): 55-64, 2006.

23 Scott NJ, Cameron VA, Raudsepp S, Lewis LK, Simpson ER, Richards AM and Ellmers LJ: Generation and characterization of a mouse model of the metabolic syndrome: Apolipoprotein $\mathrm{E}$ and aromatase double knockout mice. Am J Physiol Endocrinol Metab 302(5): E576-84, 2012.

24 Wong SK, Chin K-Y, Suhaimi FH, Fairus A and Ima-Nirwana S: Animal models of metabolic syndrome: a review. Nutr Metab 13: $65,2016$.
25 Kennedy AJ, Ellacott KLJ, King VL and Hasty AH: Mouse models of the metabolic syndrome. Dis Model Mech 3(3-4): 156-166, 2010.

26 Cleary MP: Impact of obesity on development and progression of mammary tumors in preclinical models of breast cancer. $\mathrm{J}$ Mammary Gland Biol Neoplasia 18(3-4): 333-343, 2013.

27 Choi SH, Leem J, Park S, Lee C-K, Park K-G and Lee I-K: Gemigliptin ameliorates Western-diet-induced metabolic syndrome in mice. Can J Physiol Pharmacol 95(2): 129-139, 2017.

28 Workman P, Aboagye EO, Balkwill F, Balmain A, Bruder G, Chaplin DJ, Double JA, Everitt J, Farningham DA, Glennie MJ, Kelland LR, Robinson V, Stratford IJ, Tozer GM, Watson S, Wedge SR and Eccles SA: Guidelines for the welfare and use of animals in cancer research. Br J Cancer 102(11): 1555-1577, 2010.

29 Fisher CR, Graves KH, Parlow AF and Simpson ER: Characterization of mice deficient in aromatase (ArKO) because of targeted disruption of the Cyp19 gene. Proc Natl Acad Sci USA 95(12): 6965-6970, 1998.

30 Sugiura K and Stock CC: Studies in a tumor spectrum.I: Comparison of the action of methylbis(2-chloroethyl)amine and 3-bis(2-chloroethyl)aminomethyl-4-methoxymethyl-5-hydroxy6-methylpyridine on the growth of a variety of mouse and rat tumors. Cancer 5(2): 382-402, 1952.

31 Campbell EJ, Vissers MCM, Wohlrab C, Hicks KO, Strother RM, Bozonet SM, Robinson BA and Dachs GU: Pharmacokinetic and anticancer properties of high dose ascorbate in solid tumours of ascorbate-dependent mice. Free Radic Biol Med 99: 451-462, 2016.

32 Buss LA and Dachs GU: Voluntary exercise slows breast tumor establishment and reduces tumor hypoxia in $A p o E^{-/-}$mice. $\mathrm{J}$ Appl Physiol 124(4): 938-949, 2018.

33 Yamamoto M, Patel NA, Taggart J, Sridhar R and Cooper DR: A shift from normal to high glucose levels stimulates cell proliferation in drug sensitive MCF-7 human breast cancer cells but not in multidrug resistant MCF-7/ADR cells which overproduce PKC-BII. Int J Cancer 83(1): 98-106, 1999.

34 Ewens A, Mihich E and Ehrke MJ: Distant metastasis from subcutaneously grown E0771 medullary breast adenocarcinoma. Anticancer Res 25(6 B): 3905-3915, 2005.

35 Liu BY, Soloviev I, Huang X, Chang P, Ernst JA, Polakis P and Sakanaka C: Mammary tumor regression elicited by Wnt signaling inhibitor requires IGFBP5. Cancer Res 72(6): 1568-1578, 2012.

36 Akkiprik M, Hu L, Sahin A, Hao X and Zhang W: The subcellular localization of IGFBP5 affects its cell growth and migration functions in breast cancer. BMC Cancer 9: 103, 2009.

37 Wang H, Arun BK, Wang H, Fuller GN, Zhang W, Middleton LP and Sahin AA: IGFBP2 and IGFBP5 overexpression correlates with the lymph node metastasis in $\mathrm{T} 1$ breast carcinomas. Breast J 14(3): 261-267, 2008.

38 Dethlefsen C, Højfeldt G and Hojman P: The role of intratumoral and systemic IL-6 in breast cancer. Breast Cancer Res Treat 138(3): 657-664, 2013. 